\title{
Physical Activity, Sedentariness, Eating Behaviour and Well-Being during a COVID-19 Lockdown Period in Greek Adolescents
}

\author{
Ioannis D. Morres ${ }^{1}$, Evangelos Galanis ${ }^{1}$, Antonis Hatzigeorgiadis ${ }^{1, *}$, Odysseas Androutsos ${ }^{2}$ \\ and Yannis Theodorakis ${ }^{1}$ (D) \\ 1 Department of Physical Education and Sport Science, University of Thessaly, 42100 Trikala, Greece; \\ iomorres@pe.uth.gr (I.D.M.); v.galanis@hotmail.com (E.G.); theodorakis@pe.uth.gr (Y.T.) \\ 2 Department of Nutrition and Dietetics, University of Thessaly, 42132 Trikala, Greece; oandroutsos@uth.gr \\ * Correspondence: ahatzi@pe.uth.gr; Tel.: +30-243-104-7009
}

Citation: Morres, I.D.; Galanis, E.; Hatzigeorgiadis, A.; Androutsos, O.; Theodorakis, Y. Physical Activity, Sedentariness, Eating Behaviour and Well-Being during a COVID-19 Lockdown Period in Greek Adolescents. Nutrients 2021, 13, 1449 . https://doi.org/10.3390/nu13051449

Academic Editor: Sebastian Schmid

Received: 18 March 2021

Accepted: 21 April 2021

Published: 24 April 2021

Publisher's Note: MDPI stays neutral with regard to jurisdictional claims in published maps and institutional affiliations.

Copyright: (c) 2021 by the authors. Licensee MDPI, Basel, Switzerland. This article is an open access article distributed under the terms and conditions of the Creative Commons Attribution (CC BY) license (https:// creativecommons.org/licenses/by/ $4.0 /)$.

\begin{abstract}
Adolescents' daily life has dramatically changed during the COVID-19 era due to the social restrictions that have been imposed, including closures of schools, leisure centers and sport facilities. The purpose of this study was to examine levels of well-being and mood and their relations with physical (in)activity and eating behaviors in adolescents during a lockdown period in Greece. A total of 950 adolescents (Mean Age $=14.41$ years \pm 1.63 ) participated in a web-based survey while education was conducted online and organized sport activities were interrupted. Participants showed poor well-being, insufficient physical activity levels and moderate scores of healthy eating behavior. Hierarchical regression analysis showed that, after controlling for the effect of gender and body mass index, increased physical activity and healthier eating behavior predicted better well-being ( $b=0.24, p<0.01$ and $b=0.19, p<0.01$, respectively), whereas sedentariness predicted worse well-being $(b=-0.16, p<0.01)$. Furthermore, it was revealed that days of physical activity per week was a stronger predictor of well-being than minutes of physical activity per week, and that both in-house and out-of-house physical activity were beneficial. Considering that well-being in our study was below the threshold recommended by the World Health Organization as indicative of possible depressive symptoms, measures to increase physical activity, decrease sedentariness and improve eating behavior should become a priority for communities and policy makers.
\end{abstract}

Keywords: pandemic; COVID-19; lockdown; exercise; sport; diet; mood; quality of life

\section{Introduction}

Physical activity is defined as "any bodily movement produced by skeletal muscles that results in energy expenditure" [1] and includes various subsets such as walking, cycling or sport. A subset of physical activity that is planned, structured, repetitive and purposive to fitness gains is typically called "exercise" [1]. Physical activity is a preventive/therapeutic factor towards psychological well-being components or proxies while sedentariness an adverse factor. Particularly, large-scale cross sectional studies in adults have linked physical activity to lower risk of depression [2] and sedentariness to higher risk of depression [3]. Furthermore, systematic reviews or cross sectional studies found that exercise is related with lower depression [4,5] or anxiety [6] in adult samples. Various systematic reviews of cross sectional and empirical studies in young people ( $<18$ years) have also demonstrated the positive and negative role, respectively, of physical activity and sedentariness in well-being $[7,8]$. Similarly, meta-analyses showed that physical activity in young people improves well-being components such as depression [9,10] or anxiety [11,12]. The World Health Organization (WHO) [13] guidelines for better mental health recommend physical activity of $\geq 420 \mathrm{~min} /$ week for adolescents, a larger amount of time than for adults (150-300 $\mathrm{min} /$ week). This highlights the importance of increased physical activity for better well-being in adolescents. 
However, physical activity and well-being are decreasing worldwide after the COVID19 pandemic declaration by the WHO in March 2020. Particularly, lockdown measures via social distance restrictions including closures of schools, leisure facilities and sport clubs have been repeatedly introduced to contain the spread of the SARS-CoV-2 virus but led adolescents to lower physical activity and well-being [14-19]. In spite of this rather somber reality, some encouraging findings were provided by a study conducted in China [16], which reported that even limited weekly physical activity ( $>60$ min of weekly) was linked to lower risk of deteriorated well-being in young people during the COVID-19 pandemic. In line, the WHO [13] recommends $>60 \mathrm{~min} /$ day of moderate-vigorous physical activity for better mental health in adolescents. The importance of physical activity for adolescents is highlighted further by a series of research findings in the era of COVID-19. Particularly, it has been found that physical activity lowers sedentariness and sleep disturbance [19], which are, in turn, linked to an improvement of the widely deteriorated dietary behavior $[20,21]$. The importance of addressing healthy dietary behavioral patterns should represent a constant concern. State-of-the-art outlines have reported an update for healthy eating across five continents and for Mediterranean diet, with physical activity being an inherent component $[22,23]$.

In the imposed COVID-19 lockdown period in Greece (spring 2020), adults showed decreased physical activity [24] increased depressive symptoms [25], disturbed eating behavioral patterns [26] and the lowest well-being across the 27 European Union member states [27]. In the same period, $25.9 \%$ of Greek young adults (University students) have shown increased depressive symptoms corresponding to a threshold for clinical depression [28]. Despite these dramatic changes, the prevalence of well-being, the level of physical (in) activity, the status of eating behaviors and the predictive well-being effects of physical (in) activity and eating behaviors have not been explored in Greek adolescents during a COVID-19 lockdown period. This exploration is essential, as many Greek adolescents in the pre-COVID-19 era showed poor eating behavior [29] and of the lowest well-being levels across 31 countries [30]. This exploration should thus be prioritised to update decisionmakers on effective policies for adolescents during a lockdown period, especially since $35 \%$ of the Greek families reported well-being decreases for their kids ( $<18$ years) in the first lockdown during spring 2020 [31].

Therefore, this study aimed to explore the prevalence of poor well-being, and the levels and predictive effects of physical activity and eating behavior on well-being in adolescents during the second total lockdown period (November 2020-January 2021) in Greece.

\section{Materials and Methods}

The study was conducted in accordance with the Declaration of Helsinki and received ethical approval from the University of Thessaly ethics committee (Re: 1723;/09-12-2020). An online survey was prepared using Google Forms, which allowed gathering information during the COVID-19 pandemic lockdown period. Inclusion criteria for participating in this study were age, 12-17 years, and educational level, attending the secondary Greek education (Gymnasium or Lyceum). First, a social media search was conducted with the aim to allocate groups of interest for parents (schools, institutions, parent/guardian groups or associations). The survey was subsequently communicated to such lists. In this communication parents were informed regarding the aim of the survey, the procedures, and the expected benefits. They were also reassured that participation was voluntary and they, or their children, could withdraw from the completion of the survey if they wished. Parents were subsequently asked to provide their consent for their children's participation, and finally asked to forward the survey to their children. The completion of the survey took approximately $15 \mathrm{~min}$ and was based on self-reports. Data were collected between mid-January and the beginning of February 2021. Hence, the data captures the reactions of participants in the period of the second lockdown period, when school classes were conducted online and the vast majority of sport activities for our target group were interrupted. 


\subsection{Sample}

Participants were 950 adolescents (518 boys) aged 12 to 17 years (Mage $=14.41$, $\mathrm{SD}=1.63$ ), who attended secondary education in Greece (46\% junior high school (Gymnasium), 54\% high school (Lyceum)). Based on self-reports on weight and high, the mean Body Mass Index (BMI) of participants was 21.06 (SD = 2.30). Among them, $698(73 \%)$ were members of sport clubs with mean sport experience $7.17(\mathrm{SD}=2.89)$ years, mean competitive experience $4.70(\mathrm{SD}=2.26)$ years; they were training between 3 and 6 days per week $(\mathrm{M}=4.57, \mathrm{SD}=1.43$ days $)$ for an average of $6.94(\mathrm{SD}=4.28)$ hours.

\subsection{Measures}

\subsubsection{Physical Activity and Sedentary Behavior}

The International Physical Activity Questionnaire Short Form (IPAQ-SF) [32], in particular, the Greek version previously implemented to adolescents [33], was used to assess participants' physical activity and sedentary behavior. The questionnaire assesses frequency (days/week) and duration (minutes/day) of light, moderate, and vigorous intensity physical activity, and in addition sedentary time per day. The amount of physical activity per week, per category of intensity, is calculated by multiplying the number of days/week reported by the minutes/day reported. Sedentary time is calculated by multiplying the average sedentary time per day by seven. The total physical activity per week was calculated by adding the minutes of the different intensities. Two items similar to the ones of the IPAQ-SF were added in this survey to better capture physical activity during the lockdown period; these asked participants to indicate frequency and duration of physical activity in the house and out of the house (e.g., street, park, sport venues). The scores for these additional items were calculated accordingly.

\subsubsection{Mood}

The Greek version of the 4-Dimensional Mood Scale (4DMS) [34] was used to assess participants' mood. The scale consists of 20 adjectives assessing positive energy (positive affectivity and high activation-four items), relaxation (positive affectivity and low activation-five items), negative arousal (negative affectivity and high activation-six items), and tiredness (negative affectivity and low activation-five items). Participants rated each adjective on the extent to which it generally described their mood during the previous seven days using a 5 -point Likert format $(1=$ not at all, $5=$ very much).

\subsubsection{Psychological Well-Being}

The World Health Organization Well-Being Index-5 [33] was used to assess participants' psychological well-being. This scale consists of five questions focusing on subjective quality of life based on positive mood (good spirits, relaxation), vitality (being active and waking up fresh and rested) and general interest (being interested in things). Participants were asked to indicate their levels of well-being during the previous seven days. Responses were given on a 6-point Likert scale $(0=$ at no time, $5=$ all the time), with lower scores indicating worse well-being. According to the WHO, the Well-Being Index-5 has been translated in more than 30 languages including the Greek language and can be employed for children aged 9 years old and above [33].

\subsubsection{Eating Behavior}

A slightly modified version of the Short Diet Behaviour Questionnaire for Lockdowns (SDBQ-L) [35,36] was used to address eating behavior for the study population. Following a short description of what consists healthy eating, participants asked to indicate whether (a) they were following a healthy diet, (b) they were eating more than usual, (c) they were eating on a consistent schedule, and (d) they were eating out of control. Responses were provided on a 7-point Likert scale ( $1=$ definitely no, $7=$ definitely yes $)$, with higher scores indicating better eating behavior after reversing scores for items ' $b$ ' and ' $\mathrm{d}$ '. The SDBQ-L has been previously administrated in Greek populations [35]. 


\subsubsection{Data Analysis}

Descriptive statistics, Cronbach's alpha coefficients for the scales, and correlations between all variables are presented in Table 1 . The reliability of all scales was satisfactory, ranging from 0.80 to 0.87 . Notably, first and foremost, participants' mean score on wellbeing index was close to the median point of the scale, below the WHO threshold $(<13)$, indicating a poor well-being [33]. Scores for positive moods were moderate and for negative moods moderate to low. Moderate and vigorous physical activity combined were below the $50 \%$ of the recommended by WHO physical activity. Finally, scores on healthy eating habits were moderate. Well-being was strongly, positively related to positive moods and negatively to negative moods. Physical activity and healthy eating habits showed moderate positive correlations with well-being and positive moods, and negative low correlations with negative moods. Finally, sedentary behavior showed negative correlations with well-being and positive moods and positive correlations with negative moods. 
Table 1. Descriptive statistics, Cronbach's alpha, and correlations.

\begin{tabular}{|c|c|c|c|c|c|c|c|c|c|c|c|c|c|}
\hline & \multicolumn{2}{|c|}{ Descriptives } & \multirow[t]{2}{*}{ Alpha } & \multicolumn{10}{|c|}{ Correlations } \\
\hline & $\mathbf{M}$ & SD & & 1 & 2 & 3 & 4 & 5 & 6 & 7 & 8 & 9 & 10 \\
\hline 1. Well-being & 12.63 & 5.92 & 0.84 & - & & & & & & & & & \\
\hline 2. Positive energy & 3.00 & 0.98 & 0.87 & $0.67^{* *}$ & - & & & & & & & & \\
\hline $\begin{array}{l}\text { 4. Negative } \\
\text { activation }\end{array}$ & 2.07 & 0.89 & 0.87 & $-0.45^{* *}$ & $-0.25^{* *}$ & $-0.35^{* *}$ & - & & & & & & \\
\hline 5. Tiredness & 2.03 & 0.80 & 0.85 & $-0.33^{* *}$ & $-0.17 * *$ & $-0.19^{* *}$ & $0.51 * *$ & - & & & & & \\
\hline 6. Eating behaviour & 4.45 & 1.21 & 0.75 & $0.24^{* *}$ & $0.29 * *$ & $0.13^{* *}$ & $-0.09 * *$ & $-0.09 * *$ & - & & & & \\
\hline 8. Moderate PA & 97.28 & 119.55 & - & $0.24^{* *}$ & $0.22 * *$ & $0.08^{* *}$ & $-0.07^{*}$ & -0.04 & $0.13^{* *}$ & $0.39 * *$ & - & & \\
\hline 9. Vigorous PA & 70.23 & 104.06 & - & $0.27^{* *}$ & $0.29 * *$ & 0.06 & $-0.07^{*}$ & 0.01 & $0.14^{* *}$ & $0.35 * *$ & 0.31 ** & - & \\
\hline 10. Total PA & 415.28 & 334.29 & - & $0.35^{* *}$ & $0.36^{* *}$ & $0.12 * *$ & $-0.10^{* *}$ & -0.01 & $0.16^{* *}$ & $0.87^{* *}$ & $0.70^{* *}$ & $0.64^{* *}$ & - \\
\hline 11. Sedentary time & 439.11 & 216.57 & - & $-0.24^{* *}$ & $-0.27^{* *}$ & $-0.10^{* *}$ & $0.19^{* *}$ & $0.14^{* *}$ & $-0.10^{* *}$ & $-0.15^{* *}$ & -0.03 & $-0.11^{* *}$ & $-0.14^{* *}$ \\
\hline
\end{tabular}




\section{Results}

\subsection{Preliminary Analyses}

Descriptive statistics, Cronbach's alpha coefficients for the scales and correlations between all variables are presented in Table 1 . The reliability of all scales was satisfactory, ranging from 0.80 to 0.87 . Notably, first and foremost, participants' mean score on wellbeing index was close to the median point of the scale, below the WHO threshold, indicating a poor well-being. Scores for positive moods were moderate and for negative moods moderate to low. Moderate and vigorous physical activity combined were below the 50\% of the recommended by WHO physical activity for adolescents. Finally, scores on healthy eating habits were moderate. Well-being was strongly, positively related to positive moods and negatively to negative moods. Physical activity and healthy eating habits showed moderate positive correlations with well-being and positive moods, and negative low correlations with negative moods. Finally, sedentary behavior showed negative correlations with well-being and positive moods and positive correlations with negative moods.

\subsection{Group Differences}

Two, two-way $(2 \times 2)$ multivariate analyses of variance, one for the psychometric variables (well-being and mood) and one for the behavioral variables (total physical activity, sedentary time, and healthy eating habits), were conducted to test for differences as a function of sex and sport participation. Mean scores for all subgroups are presented in Table 2.

Table 2. Mean scores for all subgroups.

\begin{tabular}{|c|c|c|c|c|c|c|c|c|}
\hline & \multicolumn{2}{|c|}{ Boys } & \multicolumn{2}{|c|}{ Girls } & \multicolumn{2}{|c|}{ Athletes } & \multicolumn{2}{|c|}{ Non-Athletes } \\
\hline & $\mathbf{M}$ & SD & $\mathbf{M}$ & SD & $\mathbf{M}$ & SD & $\mathbf{M}$ & SD \\
\hline 1. Well-being & 14.41 & 5.55 & 10.47 & 5.63 & 13.22 & 5.90 & 10.96 & 5.65 \\
\hline 2. Positive energy & 3.22 & 0.95 & 2.73 & 0.96 & 3.07 & 1.00 & 2.86 & 0.91 \\
\hline 3. Relaxation & 2.95 & 0.76 & 2.50 & 0.79 & 2.77 & 0.83 & 2.70 & 0.75 \\
\hline 4. Negative activation & 1.83 & 0.72 & 2.36 & 0.97 & 2.03 & 0.84 & 2.18 & 0.99 \\
\hline 5. Tiredness & 1.93 & 0.71 & 2.16 & 0.89 & 2.01 & 0.77 & 2.11 & 0.89 \\
\hline 6. Eating behaviour & 4.38 & 1.18 & 4.53 & 1.24 & 4.49 & 1.23 & 4.34 & 1.14 \\
\hline 7. Light PA & 281,96 & 221,42 & 205,71 & 184,62 & 267,57 & 209,79 & 190,99 & 196,03 \\
\hline 8 Moderate PA & 106,74 & 123,19 & 85,80 & 114,24 & 112,57 & 128,05 & 54,58 & 77,79 \\
\hline 9. Vigorous PA & 85,42 & 118,82 & 51,74 & 79,26 & 80,40 & 108,28 & 41,51 & 85,16 \\
\hline 10. Total PA & 474.12 & 359.28 & 343.25 & 288.00 & 460.55 & 339.34 & 287.09 & 281.84 \\
\hline 11. Sedentary time & 413.73 & 210.68 & 469.55 & 219.82 & 429.29 & 211.53 & 466.37 & 228.19 \\
\hline
\end{tabular}

M: Mean; SD: Standard Deviation; PA: Physical Activity.

Regarding the psychometric variables, the analyses showed significant multivariate effects for sex, $\mathrm{F}(5,941)=22.21, p<0.01$, partial $\eta 2=0.11$, and sport participation, $\mathrm{F}(5,941)=4.87, p<0.01$, partial $\eta 2=0.03$, and a non-significant interaction effect, $\mathrm{F}(5,941)=1.45, p=0.20$. Examination of the univariate effects for sex revealed significant differences in well-being, $\mathrm{F}(1,949)=74.99, p<0.01$, partial $\eta 2=0.07$, positive energy, $\mathrm{F}(1,949)=29.68, p<0.01$, partial $\eta 2=0.03$, relaxation, $\mathrm{F}(1,949)=55.76, p<0.01$, partial $\eta 2=0.06$, negative activation, $\mathrm{F}(1,949)=66.87, p<0.01$, partial $\eta 2=0.07$, and tiredness, $\mathrm{F}(1,949)=18.52, p<0.01$, partial $\eta 2=0.02$, with boys scoring higher than girls on well-being and positive moods, and lower on negative moods. Examination of the univariate effects for sport participation revealed significant differences in well-being, $\mathrm{F}(1,949)=18.17, p<0.01$, partial $\eta 2=0.02$, and positive energy, $\mathrm{F}(1,949)=11.40, p<0.01$, partial $\eta 2=0.01$, with athletes scoring higher than non-athletes, and non-significant effects for relaxation, $\mathrm{F}(1,949)=0.03, p=0.86$, negative activation, $\mathrm{F}(1,949)=1.72, p=0.19$, and tiredness, $\mathrm{F}(1,949)=1.21, p=0.27$.

Regarding the behavioral variables, the analyses showed significant multivariate effects for sex, $\mathrm{F}(3,942)=11.23, p<0.01$, partial $\eta 2=0.04$, and sport participation, $\mathrm{F}(3,942)=15.26, p<0.01$, partial $\eta 2=0.05$, and a non-significant interaction effect, $F(3,942)=0.78, p=0.51$. Examination of the univariate effects for sex revealed signif- 
icant differences in total physical activity, $\mathrm{F}(1,948)=21.56, p<0.01$, partial $\eta 2=0.02$, and sedentary time, $\mathrm{F}(3,942)=9.46, p<0.01$, partial $\eta 2=0.01$, with boys scoring higher in physical activity and lower on sedentary time, and a non-significant effect on healthy eating habits, $\mathrm{F}(1,948)=1.66, p=0.20$. Examination of the univariate effects for sport participation revealed significant differences in physical activity, $\mathrm{F}(1,948)=43.95, p<0.01$, partial $\eta 2=0.04$, and healthy eating habits, $\mathrm{F}(1,948)=3.89, p<0.01$, partial $\eta 2=0.01$, with athletes scoring higher than non-athletes, and a non-significant effect on sedentary time, $\mathrm{F}(1,948)=3.54, p=0.06$.

\subsection{Prediction of Well-Being}

Three sets of hierarchical regressions analyses were performed to examine the predictive strength of the behavioral variables for well-being, and the specifics of physical activity in predicting well-being. In all analyses, sex and BMI were entered in the first step to account for sex differences and the effect of BMI on well-being, which were identified in the preliminary analyses.

In the first hierarchical analysis, total physical activity, sedentary time and healthy eating habits were entered in the second step of the analysis. In the first step of the analysis, sex and BMI predicted $13 \%$ of the well-being variance, with both predictors being significant $(b=-0.34, p<0.01$ and $b=-0.15, p<0.01$, respectively). In the second step, the introduction of the behavioral variables raised the prediction to $27.5 \%$ of the variance. All predictors were significant; among the behavior variables, total physical activity was the stronger $(b=0.24, p<0.01)$, followed by eating habits $(b=0.19, p<0.01)$ and sedentary time $(b=-0.15, p<0.01)$. The results of this regression analysis are presented in Table 3 .

Table 3. Prediction of well-being from behavioral variables.

\begin{tabular}{|c|c|c|c|c|}
\hline & Beta & $\mathbf{t}$ & $\mathbf{R}^{2}$ & $\mathbf{F}$ \\
\hline step 1 & & & 13 & $(2,914)=68.40^{* *}$ \\
\hline Sex & -0.33 & $-10.95^{* *}$ & & \\
\hline BMI & -0.15 & $-4.89^{* *}$ & & \\
\hline step 2 & & & 27.5 & $(5,914)=68.96^{* *}$ \\
\hline Sex & -0.27 & $-9.54^{* *}$ & & \\
\hline BMI & -0.09 & $-3.28^{* *}$ & & \\
\hline Total PA & 0.24 & $8.03^{* *}$ & & \\
\hline Sedentary time & -0.16 & $-5.49^{* *}$ & & \\
\hline Eating behavior & 0.19 & $6.57^{* *}$ & & \\
\hline
\end{tabular}

The purpose of the second regression was to identify (a) the role of physical activity intensity and (b) whether frequency (i.e., number of days/week) or duration (total number of minutes/week) of physical activity were stronger predictors of well-being. Thus, in this hierarchical analysis, days and total time of light, moderate and vigorous physical activity were entered in the second step of the analysis. In the second step of the model, the introduction of the physical activity raised the prediction from $13.1 \%$ to $23.4 \%$ of the variance. Interestingly, the frequency of light, moderate and vigorous physical activity were all significant predictors of well-being, while the duration was not significant for any of them. Similar coefficients were revealed for all types of activity; for vigorous, $b=0.12$, $p<0.01$, for moderate, $(b=0.10, p<0.01)$, and for light, $(b=0.14, p<0.01)$. The results of this regression analysis are presented in Table 4. 
Table 4. Prediction of well-being from physical activity intensity, frequency and duration.

\begin{tabular}{ccccc}
\hline & Beta & $\mathbf{t}$ & $\mathbf{R}^{\mathbf{2}}$ & $\mathbf{F}$ \\
\hline step 1 & & & 13.1 & $(2,915)=68.88^{* *}$ \\
Sex & -0.33 & $-10.95^{* *}$ & & \\
BMI & -0.15 & $-4.89^{* *}$ & & \\
step 2 & & & 23.4 & $(8,914)=34.55^{* *}$ \\
Sex & -0.26 & $-8.80^{* *}$ & & \\
BMI & -0.10 & $-3.57^{* *}$ & & \\
Light PA-days & 0.13 & $4.011^{* *}$ & & \\
Light PA-time & 0.03 & 0.92 & & \\
Moderate PA-days & 0.10 & $2.55^{*}$ & & \\
Moderate PA-time & 0.04 & 1.25 & & \\
Vigorous PA-days & 0.12 & $2.87^{* *}$ & & \\
Vigorous PA-time & 0.01 & 0.36 & & \\
${ }^{*} p<05 ;{ }^{* *} p<0.01$; BMI: Body Mass Index; PA: Physical Activity. & &
\end{tabular}

The purpose of the third regression analysis was to identify the role of physical activity location. Thus, in this hierarchical analysis, days of in-house physical activity and days of out-of-house physical activity were entered in the second step of the analysis. In the second step, the introduction of the physical activity raised the prediction from $13.1 \%$ to $22.6 \%$ of the variance. Both variables were significant predictors of well-being, with out-of-house physical activity $(b=0.23, p<0.01)$ being stronger than in-house physical activity $(b=0.17$, $p<0.01)$. The results of this regression analysis are presented in Table 5.

Table 5. Prediction of well-being from physical activity location.

\begin{tabular}{ccccc}
\hline & Beta & $\mathbf{t}$ & $\mathbf{R}^{2}$ & $\mathbf{F}$ \\
\hline step 1 & & & 13.1 & $(2,913)=68.89$ \\
Sex & -0.34 & $10.99^{* *}$ & & \\
BMI & -0.15 & $4.92^{* *}$ & & \\
step 2 & & & 22.6 & $(4,911)=66.43$ \\
Sex & -0.27 & $9.10^{* *}$ & & \\
BMI & -0.11 & $3.79^{* *}$ & & \\
Home PA & 0.17 & $5.59^{* *}$ & & \\
Out-of-home PA & 0.23 & $7.41^{* *}$ & & \\
${ }^{*} p<0.01$; BMI: Body Mass Index; PA: Physical Activity. & &
\end{tabular}

\section{Discussion}

The present study aimed primarily at identifying relationships between physical activity, sedentariness, eating behavior and well-being among adolescents during a lockdown period, while school classes were delivered on-line and organized sport activities were interrupted. The most striking result was the worryingly low levels of well-being reported by participants. The average score on well-being, and accordingly the score of $49.5 \%$ of the participants, was below the threshold (total score $<13$ ) identified by the $\mathrm{WHO}$ as requiring inspection for depressive symptoms [33]. Similarly, 48.3\% of Australian adolescents in a COVID-19 lockdown showed mental distress corresponding to a probable mental illness [37]. Poor well-being components have also been recorded in Philippines where $27 \%$ to $40 \%$ of young people aged 12 to 21 years old showed increased levels of anxiety and/or depressive symptoms [38].

The signaling effects of the COVID-19 on Greek adolescents have been identified during the previous lockdown period, in spring 2020. In a study with parents, it was reported that the psychological health of children $(<18$ years) was considerably affected in 35\% of the Greek families [31]. These findings are similar to the findings from Canada where $35.7 \%$ of parents were found to be experiencing high levels of anxiety due to COVID19 [39]. Similarly discouraging findings were found for young people and/or parents in the previous lockdown period in Italy $[40,41]$ or in Greece; for example, University students in 
Greece showed considerable reduction of mental health, including increased anxiety and depressive symptoms corresponding to a threshold for clinical depression [28].

Encouragingly, physical activity and healthy patterns of eating behavior were positively related with participants' positive mood dimensions and well-being. Furthermore, adolescents who used to participate in organized sport reported higher levels of physical activity and better well-being compared to those not participating in organized sport, above the threshold suggested by the WHO for better well-being but still low. Despite the interruption of sport due to the restrictions, which should have had a more dramatic impact on athletes' physical activity and subsequently well-being, athletes managed to maintain a more active lifestyle and better well-being. Various studies have repeatedly related physical activity with better well-being components including lower depression and anxiety symptoms in adolescents $[11,12]$ and adults $[4,6]$. In light of this repeated positive evidence, the WHO [13] guidelines recommend adolescents perform physical activity of $60 \mathrm{~min} /$ day ( $\geq 420 \mathrm{~min} /$ week) for better mental health. Physical activity of participants in the present study was below the recommended by the WHO levels, nevertheless, was the stronger predictor of well-being, even after controlling for the effects of sex and BMI that were also found to influence well-being. The beneficial effects of physical activity for mental health during the COVID-19 pandemic have been also evidenced in young Chinese persons by the authors of [16], who reported that physical activity for $>60 \mathrm{~min} /$ week was linked to lower risk of deteriorated well-being.

Healthy eating emerged as a secondary significant predictor of better well-being. Indeed, healthy eating in the COVID-19 pandemic is linked to improved well-being components such as lower depressive and anxious symptoms [42]. However, eating behavioral patterns have deteriorated in the COVID-19 pandemic (e.g., number of main meals or snacks between meals, eating out of control or type of food) [36]. Furthermore, data from Cyprus for the pre- and post-lockdown period due to COVID-19 reported a significant increase of young people (5-14 years old) consuming food items containing sugar [18]. Initiatives towards healthier eating behavior at home are thus essential, and relevant updates have been recently published for further action. In particular, the importance of addressing healthy dietary behavioral patterns represents a constant concern. State-of-the-art outlines have reported an update for healthy eating across five continents and for Mediterranean diet, with physical activity being an inherent component [22,23]. Such initiatives, however, seem to be potentially challenging due to adolescents' poor well-being. Despite this somber reality, the multiple benefits of physical activity reveal promising perspectives towards healthier eating. In particular, physical activity lowers sedentariness and sleep disturbance [19], which are, in turn, linked to less deteriorated dietary behavior [20,21]. This beneficial sequence seems to be supported, although not examined, in our study by the correlations amongst house-based physical activity, better eating behavior and improved well-being.

In contrast to physical activity and healthy eating behavior, sedentary time predicted well-being deterioration in our study. This is an unsurprising finding because sedentary time has been repeatedly associated with deteriorated well-being components (e.g., depression) in large-scale epidemiological studies across adolescents $[7,8]$ and adult populations [3]. Moreover, adolescents living in COVID-19 lockdowns have demonstrated increased sedentariness and low well-being [14-19]; sitting time in particular has dramatically increased [36] and clearly related with deteriorated well-being components including sleeping patterns and depressive and anxiety symptoms [43]. Tackling sedentary time in Greek adolescents during the COVID-19 pandemic and involved lockdowns should thus be prioritized to decrease sitting time modalities such as the widespread screen time. Towards this direction, it would be interesting to consider the potentially detrimental effects of online schooling on physical activity, and address to school directors and policy makers the importance of physically active breaks between classes in the daily schedule.

A final issue deserving consideration is the relevant contribution of physical activity modalities towards the prediction of well-being. The first notable point is that frequency of physical activity (days/week) was a stronger predictor of duration (total minutes/week), 
for all physical activity intensity modes. The emerged importance of frequency in physical activity participation has significant implications for how the recommendations for physical activity that should be prioritized, at least during the pandemic times. Small bouts of physical activity may increase overall frequency (days/week) without increasing risks of injuries, which are typically linked to accumulated physical activity volume. In line with this, a recent study has reported the contribution of small bouts to increased frequency of physical activity among children [44]. The second issue is that out-of-house physical activity was a stronger predictor of well-being; yet, after accounting for that effect, inhouse physical activity was still a significant predictor. Even though the superiority of the out-of-house activity is a reasonable finding, that in-house physical activity during a lockdown time, where the chances of going out of the house are limited, highlights the unique impact that physical activity per se, not combined with going out, can have on well-being. Therefore, the promotion of ideas and innovative ways to exercise in the house should be particularly emphasized during lockdown periods.

\section{Limitations}

The findings of the current study should be considered under the light of certain limitations. First, the cross-sectional design of the study does not allow causal conclusions with regard to the statistical prediction of well-being from physical activity, sedentariness and eating habits; second, the reliance on self-reported behavioral measures, including physical activity, sedentary behavior and eating habits, which are dependent on memory and, in cases, liable to social desirability; third, the consideration of additional, to gender and BMI, control variables that may have influenced the results; and finally the recruitment of the sample through the social media, which does not allow calculation of response rates and limits the participation of people without access to the internet or non-users of social media, thus limiting the generalizability of the findings. These limitations, despite being typical in large scale surveys, and in particular web-based surveys, suggest that our findings should be interpreted with caution.

\section{Conclusions}

Action needs to be taken by both researchers and policy makers to enhance well-being in Greek adolescents by providing guidance and opportunities for increased physical activity, decreased sedentary time and healthier eating behaviors. Such action is of high priority as $50 \%$ of our sample showed levels of well-being below the general population norms. These levels signify according to the $\mathrm{WHO}$ the need to subsequently investigate for potential depression; thus, prompt research is warranted to address this important health risk that emerged during the pandemic. Considering evidence suggesting that Greek adolescents' physical activity and well-being are among the lowest in Europe even before the emergence of the pandemic [30], initiatives targeting the improvement of healthy habits should be supported hereafter with consistency.

Author Contributions: Conceptualization, Y.T. and I.D.M.; methodology, A.H. and O.A.; formal analysis, E.G. and A.H.; writing-original draft preparation, I.D.M. and E.G.; writing-review and editing, A.H., O.A. and Y.T. All authors have read and agreed to the published version of the manuscript.

Funding: This research received no external funding.

Institutional Review Board Statement: The study was conducted according to the guidelines of the Declaration of Helsinki, and approved by the Ethics Committee of the Department of Physical Education \& Sport Science, of the University of Thessaly (Re: 1723/09-12-2020).

Informed Consent Statement: Informed consent was obtained from parents of all subjects involved in the study.

Data Availability Statement: Data are available upon request from the first author.

Conflicts of Interest: The authors declare no conflict of interest. 


\section{References}

1. Caspersen, C.J.; Powell, K.E.; Christenson, G. Physical activity, exercise and physical fitness: Definitions and distinctions for health-related research. Public Health Rep. 1985, 100, 126-131.

2. Harris, A.H.; Cronkite, R.; Moos, R. Physical activity, exercise coping, and depression in a 10-year cohort study of depressed patients. J. Affect. Disord. 2006, 93, 79-85. [CrossRef]

3. Zhai, L.; Zhang, Y.; Zhang, D. Sedentary behaviour and the risk of depression: A meta-analysis. Br. J. Sports Med. 2015, 49, 705-709. [CrossRef]

4. Morres, I.D.; Hatzigeorgiadis, A.; Stathi, A.; Comoutos, N.; Arpin-Cribbie, C.; Krommidas, C.; Theodorakis, Y. Aerobic exercise for adult patients with major depressive disorder in mental health services: A systematic review and meta-analysis. Depress. Anxiety 2019, 36, 39-53. [CrossRef]

5. Morres, I.D.; Hatzigeorgiadis, A.; Krommidas, C.; Comoutos, N.; Sideri, E.; Ploumpidis, D.; Economou, M.; Papaioannou, A.; Theodorakis, Y. Objectively measured physical activity and depressive symptoms in adult outpatients diagnosed with major depression. Clinical perspectives. Psychiatry Res. 2019, 280, 112489. [CrossRef] [PubMed]

6. Wipfli, B.M.; Rethorst, C.D.; Landers, D.M. The anxiolytic effects of exercise: A meta-analysis of randomized trials and doseresponse analysis. J. Sport Exerc. Psychol. 2008, 30, 392-410. [CrossRef] [PubMed]

7. Burkhardt, J.; Brennan, C. The effects of recreational dance interventions on the health and well-being of children and young people: A systematic review. Arts Health 2012, 4, 148-161. [CrossRef]

8. Biddle, S.J.H.; Asare, M. Physical activity and mental health in children and adolescents: A review of reviews. Br. J. Sports Med. 2011, 45, 886-895. [CrossRef] [PubMed]

9. Carter, T.; Morres, I.D.; Meade, O.; Callaghan, P. The Effect of Exercise on Depressive Symptoms in Adolescents: A Systematic Review and Meta-Analysis. J. Am. Acad. Child Adolesc. Psychiatry 2016, 55, 580-590. [CrossRef]

10. Wegner, M.; Amatriain-Fernández, S.; Kaulitzky, A.; Murillo-Rodriguez, E.; Machado, S.; Budde, H. Systematic Review of Meta-Analyses: Exercise Effects on Depression in Children and Adolescents. Front. Psychiatry 2020, 11, 81. [CrossRef]

11. Philippot, A.; Meerschaut, A.; Danneaux, L.; Smal, G.; Bleyenheuft, Y.; De Volder, A.G. Impact of Physical Exercise on Symptoms of Depression and Anxiety in Pre-adolescents: A Pilot Randomized Trial. Front. Psychol. 2019, 10, 1820. [CrossRef] [PubMed]

12. Carter, T.; Pascoe, M.; Bastounis, A.; Morres, I.D.; Callaghan, P.; Parker, A.G. The effect of physical activity on anxiety in children and young people: A systematic review and meta-analysis. J. Affect. Disord. 2021, 285, 10-21. [CrossRef] [PubMed]

13. World Health Organization. WHO Guidelines on Physical Activity and Sedentary Behaviour; WHO: Geneva, Switzerland, 2020.

14. Vanderloo, L.M.; Carsley, S.; Aglipay, M.; Cost, K.T.; Maguire, J.; Birken, C.S. Applying Harm Reduction Principles to Address Screen Time in Young Children Amidst the COVID-19 Pandemic. J. Dev. Behav. Pediatrics 2020, 41, 335-336. [CrossRef]

15. Margaritis, I.; Houdart, S.; El Ouadrhiri, Y.; Bigard, X.; Vuillemin, A.; Duché, P. How to deal with COVID-19 epidemic-related lockdown physical inactivity and sedentary increase in youth? Adaptation of Anses' benchmarks. Arch. Public Health 2020, 78, 52. [CrossRef] [PubMed]

16. Qin, Z.; Shi, L.; Xue, Y.; Lin, H.; Zhang, J.; Liang, P.; Lu, Z.; Wu, M.; Chen, Y.; Zheng, X.; et al. Prevalence and Risk Factors Associated with Self-reported Psychological Distress among Children and Adolescents During the COVID-19 Pandemic in China. JAMA Netw. Open 2021, 4, e2035487. [CrossRef] [PubMed]

17. Stavridou, A.; Stergiopoulou, A.-A.; Panagouli, E.; Mesiris, G.; Thirios, A.; Mougiakos, T.; Troupis, T.; Psaltopoulou, T.; Tsolia, M.; Sergentanis, T.N.; et al. Psychosocial consequences of COVID-19 in children, adolescents and young adults: A systematic review. Psychiatry Clin. Neurosci. 2020, 74, 615-616. [CrossRef]

18. Konstantinou, C.; Andrianou, X.D.; Constantinou, A.; Perikkou, A.; Markidou, E.; Christophi, C.A.; Makris, K.C. Exposome changes in primary school children following the wide population non-pharmacological interventions implemented due to COVID-19 in Cyprus: A national survey. EClinicalMedicine 2021, 32, 100721. [CrossRef] [PubMed]

19. Bates, L.C.; Zieff, G.; Stanford, K.; Moore, J.B.; Kerr, Z.Y.; Hanson, E.D.; Barone Gibbs, B.; Kline, C.E.; and Stoner, L. COVID-19 Impact on Behaviors across the 24-Hour Day in Children and Adolescents: Physical Activity, Sedentary Behavior, and Sleep. Children 2020, 7, 138. [CrossRef]

20. Du, C.; Zan, M.C.H.; Cho, M.J.; Fenton, J.I.; Hsiao, P.Y.; Hsiao, R.; Keaver, L.; Lai, C.-C.; Lee, H.; Ludy, M.-J.; et al. Health Behaviors of Higher Education Students from 7 Countries: Poorer Sleep Quality during the COVID-19 Pandemic Predicts Higher Dietary Risk. Clocks Sleep 2021, 3, 12-30. [CrossRef]

21. Ruiz-Roso, M.B.; de Carvalho Padilha, P.; Mantilla-Escalante, D.C.; Ulloa, N.; Brun, P.; Acevedo-Correa, D.; Arantes Ferreira Peres, W.; Martorell, M.; Aires, M.T.; de Oliveira Cardoso, L.; et al. Covid-19 Confinement and Changes of Adolescent's Dietary Trends in Italy, Spain, Chile, Colombia and Brazil. Nutrients 2020, 12, 1807. [CrossRef]

22. Fernandez, M.L.; Raheem, D.; Ramos, F.; Carrascosa, C.; Saraiva, A.; Raposo, A. Highlights of Current Dietary Guidelines in Five Continents. Int. J. Environ. Res. Public Health 2021, 18, 2814. [CrossRef]

23. Serra-Majem, L.; Tomaino, L.; Dernini, S.; Berry, E.M.; Lairon, D.; Ngo de la Cruz, J.; Bach-Faig, A.; Donini, L.M.; Me-dina, F.-X.; Belahsen, R.; et al. Updating the Mediterranean Diet Pyramid towards Sustainability: Focus on Environmental Concerns. Int. J. Environ. Res. Public Health 2020, 17, 8758. [CrossRef] [PubMed]

24. Bourdas, D.I.; Zacharakis, E.D. Impact of COVID-19 Lockdown on Physical Activity in a Sample of Greek Adults. Sports 2020, 8 , 139. [CrossRef] [PubMed] 
25. Parlapani, E.; Holeva, V.; Voitsidis, P.; Blekas, A.; Gliatas, I.; Porfyri, G.N.; Golemis, A.; Papadopoulou, K.; Dimitriadou, A.; Chatzigeorgiou, A.F.; et al. Psychological and Behavioral Responses to the COVID-19 Pandemic in Greece. Front. Psychiatry 2020, 11, 821. [CrossRef] [PubMed]

26. Papandreou, C.; Arija, V.; Aretouli, E.; Tsilidis, K.K.; Bulló, M. Comparing eating behaviours, and symptoms of depression and anxiety between Spain and Greece during the COVID-19 outbreak: Cross-sectional analysis of two different confinement strategies. Eur. Eat. Disord. Rev. J. Eat. Disord. Assoc. 2020, 28, 836-846. [CrossRef] [PubMed]

27. European Foundation for the Improvement of Living Working Conditions. Living, Working and COVID-19: First Findings, April 2020; Publications Office of the European Union: Luxembourg, 2020. Available online: https:/ /www.eurofound.europa.eu/ publications/report/2020/living-working-and-covid-19-first-findings-april-2020 (accessed on 24 April 2020).

28. Kaparounaki, C.K.; Patsali, M.E.; Mousa, D.-P.V.; Papadopoulou, E.V.K.; Papadopoulou, K.K.K.; Fountoulakis, K.N. University students' mental health amidst the COVID-19 quarantine in Greece. Psychiatry Res. 2020, 290, 113111. [CrossRef]

29. World Health Organization. Spotlight on Adolescent Health and Well-Being; Findings from the 2017/2018 Health Behaviour in School-Aged Children (HBSC) Survey in Europe and Canada; WHO Regional Office for Europe: Copenhagen, Denmark, 2020.

30. Bruckauf, Z. Adolescents' Mental Health; United Nations: New York, NY, USA, 2017.

31. Magklara, K.; Lazaratou, H.; Barbouni, A.; Poulas, K.; Farsalinos, K. Impact of COVID-19 pandemic and lockdown measures on mental health of children and adolescents in Greece. medRxiv 2020. [CrossRef]

32. Craig, C.L.; Marshall, A.L.; Sjöström, M.; Bauman, A.E.; Booth, M.L.; Ainsworth, B.E.; Pratt, M.; Ekelund, U.; Yngve, A.; Sallis, J.F. International physical activity questionnaire: 12-country reliability and validity. Med. Sci. Sports Exerc. 2003, 35, 1381-1395. [CrossRef]

33. World Health Organization. Wellbeing Measures in Primary Health Care/the DEPCARE Project: Report on a WHO Meeting, Stockholm, Sweden, 12-13 February 1998; World Health Organization: Geneva, Switzerland, 1998.

34. Huelsman, T.J.; Nemanick, R.C.; Munz, D.C. Scales to Measure Four Dimensions of Dispositional Mood: Positive Energy, Tiredness, Negative Activation, and Relaxation. Educ. Psychol. Meas. 1998, 58, 804-819. [CrossRef]

35. Ammar, A.; Trabelsi, K.; Brach, M.; Chtourou, H.; Boukhris, O.; Masmoudi, L.; Bouaziz, B.; Bentlage, E.; How, D.; Ahmed, M.; et al. Effects of home confinement on mental health and lifestyle behaviours during the COVID-19 outbreak: Insight from the "ECLB-COVID19" multi countries survey. medRxiv 2020. [CrossRef]

36. Ammar, A.; Brach, M.; Trabelsi, K.; Chtourou, H.; Boukhris, O.; Masmoudi, L.; Bouaziz, B.; Bentlage, E. Effects of COVID-19 Home Confinement on Eating Behaviour and Physical Activity: Results of the ECLB-COVID19 International Online Survey. Nutrients 2020, 12, 1583. [CrossRef]

37. Li, S.H.; Beames, J.R.; Newby, J.M.; Maston, K.; Christensen, H.; Werner-Seidler, A. The impact of COVID-19 on the lives and mental health of Australian adolescents. medRxiv 2020. [CrossRef]

38. Tee, M.L.; Tee, C.A.; Anlacan, J.P.; Aligam, K.J.G.; Reyes, P.W.C.; Kuruchittham, V.; Ho, R.C. Psychological impact of COVID-19 pandemic in the Philippines. J. Affect. Disord. 2020, 277, 379-391. [CrossRef] [PubMed]

39. McCormack, G.R.; Doyle-Baker, P.K.; Petersen, J.A.; Ghoneim, D. Parent anxiety and perceptions of their child's physical activity and sedentary behaviour during the COVID-19 pandemic in Canada. Prev. Med. Rep. 2020, 20, 101275. [CrossRef] [PubMed]

40. Cusinato, M.; Iannattone, S.; Spoto, A.; Poli, M.; Moretti, C.; Gatta, M.; Miscioscia, M. Stress, Resilience, and Well-Being in Italian Children and Their Parents during the COVID-19 Pandemic. Int. J. Environ. Res. Public Health 2020, 17, 8297. [CrossRef] [PubMed]

41. Maugeri, G.; Castrogiovanni, P.; Battaglia, G.; Pippi, R.; D'Agata, V.; Palma, A.; Di Rosa, M.; Musumeci, G. The impact of physical activity on psychological health during Covid-19 pandemic in Italy. Heliyon 2020, 6, e04315. [CrossRef] [PubMed]

42. Chi, X.; Liang, K.; Chen, S.-T.; Huang, Q.; Huang, L.; Yu, Q.; Jiao, C.; Guo, T.; Stubbs, B.; Hossain, M.M.; et al. Mental health problems among Chinese adolescents during the COVID-19: The importance of nutrition and physical activity. Int. J. Clin. Health Psychol. IJCHP 2020, 100218. [CrossRef]

43. Lu, C.; Chi, X.; Liang, K.; Chen, S.-T.; Huang, L.; Guo, T.; Jiao, C.; Yu, Q.; Veronese, N.; Soares, F.C.; et al. Moving More and Sitting Less as Healthy Lifestyle Behaviors are Protective Factors for Insomnia, Depression, and Anxiety Among Adolescents During the COVID-19 Pandemic. Psychol. Res. Behav. Manag. 2020, 13, 1223-1233. [CrossRef]

44. Brooke, H.L.; Atkin, A.J.; Corder, K.; Brage, S.; van Sluijs, E.M.F. Frequency and duration of physical activity bouts in school-aged children: A comparison within and between days. Prev. Med. Rep. 2016, 4, 585-590. [CrossRef] 